\title{
Numerical Analysis and Geometry Optimisation of Vertical Vane of Room Air-conditioner
}

\author{
Abdulkareem Sh. Mahdi Al-Obaidi ${ }^{1, *}$, Chan Wei Yin ${ }^{1}$, Felicia Wong Yen Myan ${ }^{1}$ and Yan \\ Yao Chen ${ }^{1}$ \\ ${ }^{1}$ School of Engineering, Faculty of Built Environment, Engineering, Technology \& Design, Taylor's \\ University, Subang Jaya, Selangor DE, Malaysia
}

\begin{abstract}
Vertical vanes of room air-conditioners are used to control and direct cold air. This paper aims to study vertical vane as one of the parameters that affect the efficiency of dissipating cold air to a given space. The vertical vane geometry is analysed and optimised for lower production cost using CFD. The optimised geometry of the vertical vane should have the same or increased efficiency of dissipating cold air and have lesser mass compared to the existing original design. The existing original design of vertical vane is simplified and analysed by using ANSYS Fluent. Efficiency of wind direction is define as how accurate the direction of airflow coming out from vertical vane. In order to calculate the efficiency of wind direction, $15^{\circ}$ and $30^{\circ}$ rotation of vertical vane inside room airconditioner are simulated. The efficiency of wind direction for $15^{\circ}$ rotation of vertical vane is $57.81 \%$ while efficiency of wind direction for $30^{\circ}$ rotation of vertical vane is $47.54 \%$. The results of the efficiency of wind direction are used as base reference for parametric study. The parameters investigated for optimisation of vertical vane are focused at length of long span, tip chord and short span. The design of $15 \%$ decreased in vane surface area at tip chord is the best optimised design of vertical vane because the efficiency of wind direction is the highest as $60.32 \%$.
\end{abstract}

\section{Introduction}

Vertical vane is part of air-conditioners that can set horizontal air flow direction to left or right. Currently, vertical vane is rarely been studied as part of parameters that affect the efficiency of dissipating cold air to a given space. Most studies and researches have been done on horizontal louver's parameters that will affect the performance of air-conditioners. However, the studies that investigate the horizontal louvers can be used as reference for vertical vanes since the boundary conditions for both are the same in CFD simulation. With the assist of CFD software, geometries changes of vertical vane can be analysed without wasting money to manufacture it [1]. Moreover, CFD software can save time by simulating the case study instead of conducting physical testing with equipment such as wind tunnel. Panasonic is one of the companies that concern about any improvement can be made from design of vertical vane.

* Corresponding author: abdulkareem.mahdi@taylors.edu.my 
Nakanishi et al. [2] investigated the air flow passing through louver. The change of louver angle that cause pressure is studied. Three different louver models respectively with louver angle of $45^{\circ}, 35^{\circ}$ and $25^{\circ}$ were tested. Laser Doppler Velocimetry (LDV) and smoke wire methods have been conducted to visualise the pattern and velocity of airflow. After that, CFD++ software is used to simulate the result. The result of experiment and CFD were compared where the compressible Navier-Stokes equation and k- $\varepsilon$ turbulence model are used in CFD simulation. The CFD could have good agreement with the experimental result from LDV and smoke wire methods. It is concluded that the larger the louver angle, the larger the vortexes. The velocity of airflow is increased due to the narrowed flow path by large vortexes. As a result, the pressure loss is increased with larger louver angle.

A similar study from Noh et al. [3] was conducted which was based on the research of Nakanishi et al. [2]. This research investigates how the shape of airfoil and flat louver affect the pressure drop across weather hood. Moreover, different angles of attack which were $25^{\circ}, 35^{\circ}$ and $45^{\circ}$ were studied at both shape and flat louvers. The numerical method was conducted by using SolidWorks Flow Simulation software. Since the inlet velocity of airflow did not reach $0.3 \mathrm{Ma}$ (Mach number), incompressible flow was applied [4]. Incompressible flow was applied and standard $k-\varepsilon$ turbulence model were used in CFD simulation. It is concluded that higher louver angle will induce greater pressure drop due to the increase in velocity of airflow. Other than that, airfoil louvers show less pressure drop compared to the flat louvers. In result, airfoil louvers with louver angle of $25^{\circ}$ contributed the lowest pressure drop compared to flat louvers and other louver angles.

CFD used as reliable tool to predict the indoor airflow in recent years [5-8]. For instance, Karimipanah et al. [6] conducted several times of researches about the indoor air quality and comfort conditions in classroom. The initiative of investigating indoor air quality is due to the reason of caring the pupil's health and those children who have allergy or asthma. In this research, Karimipanah et al. compared the results calculated by RNG (Re-Normalisation Group) turbulence model and standard $\mathrm{k}-\varepsilon$ turbulence model respectively with measured result. It is concluded that RNG turbulence model can simulate velocity of airflow with higher agreement with measured result. In contrast, standard k- $\varepsilon$ turbulence model shows better capability of predicting the temperature distribution over the classroom compared to RNG turbulence model. This research gives a good idea of choosing suitable turbulence model to get desired simulation result which is approximated to measured result closely.

Sadrizadeh and Holmberg [9] conducted a very detail study about different type of turbulence models applied for indoor airflow prediction. There is nine type of turbulence models are analysed and validated with the experimental result. The experiment is carried out in a box-shaped wind tunnel. A mannequin was built to model a people sitting inside a room (wind tunnel). The locations of measured temperature and velocity were at both; front of and behind the, mannequin. Most of the Reynolds averaged Navier-Strokes approach (RANS) were selected to apply in the CFD simulation in this study. RNG k-E (Re-normalisation Group) is the best prediction of velocity of airflow out of RANS turbulence models.

This research aims to analyse and optimise the geometry of the vertical vane in order to minimise its mass for lower production cost. Besides that, optimising geometry of vertical vane shall not bring negative impact to the performance and efficiency. Firstly, the method of defining the efficiency for simplified vertical vane is studied and analysed. Next, different geometries and dimensions of vertical vane will be analysed using ANSYS Fluent ver. 18 (CFD software) for a given set of flow conditions. The research process will be follow by optimisation to select the vertical vane shape which should have same efficiency of dissipating cold air compared to the existing original vertical vane. 


\section{Research methodology}

The method of doing numerical analysis of vertical vane is written and explained in this section. Besides that, the calculation of efficiency for vertical vane is defined.

\subsection{D CAD Modelling}

The geometry of existing original design of vertical vane is studied and the assumptions of $3 \mathrm{D}$ CAD modelling is made for geometry clean-up. The simplified vertical vane is drawn by using SolidWorks ver. 2016. The existing original design and simplified vertical vane is shown in Fig. 1. The simplified vertical vane is redrawn based on a few assumptions made.

Firstly, the tip of vertical vane where it attached to air-conditioner is assumed no air flow into the hole. The reason is the tip part will later be locked with a nut and the volume will be filled after assembly. Thus, the tip of vertical vane is simplified to have filled volume. Besides that, the hook of vertical vane where the connecting bar attached to is simplified to become fillet instead of having irregular shape of hook. Changing the geometry of hook will not affect the air flow that much but it helps to reduce complexity of geometry in CFD simulation afterward. The circumference length of simplified vertical vane follows exactly the dimension of existing original design. For the upper surface of vertical vane, it is simplified to have same thickness as lower surface. The reason is the thickness of vertical vane contributes very small effect to velocity of air flow.

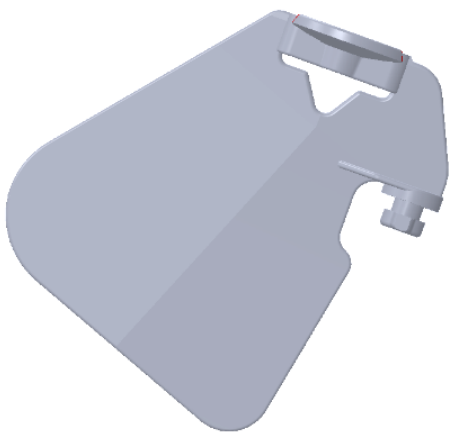

(a)

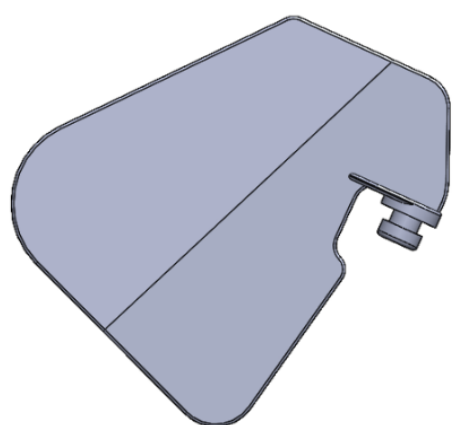

(b)

Fig. 1. Vertical vane (a) existing original design (b) simplified.

\subsection{Numerical modelling}

\subsubsection{Geometry modelling}

Geometry model for CFD is based on a condition that air-conditioner is installed in a 6 $\mathrm{m} \times 5 \mathrm{~m} \times 3.5 \mathrm{~m}$ of room. Usually, the fluid domain should build with 5 meter square base with height of $3.5 \mathrm{~m}$ for this model. However, back flow of air is not the outcome that wanted to focus in simulation. Thus, the fluid domain drawn for this model is much bigger than size of the room. It can increase the accuracy of simulated result. Shell of room air-conditioner is drawn and vertical vanes are placed inside the shell. Following by that, a half sphere shape of fluid domain is drawn and shown in Fig. 2. The half sphere is drawn with $10 \mathrm{~m}$ diameter.

Two different rotations of simplified vertical vane are studied. First case is to simulate the $0^{\circ}$ of vertical vane in Fig. 3 (a) and the second case is to simulate the $30^{\circ}$ angle of 
vertical vane in Fig. 3 (b). The case of $0^{\circ}$ angle of vertical vane is used as base reference for meshing size. The $30^{\circ}$ is the maximum rotation that allows rotating for the product of room air-conditioner.

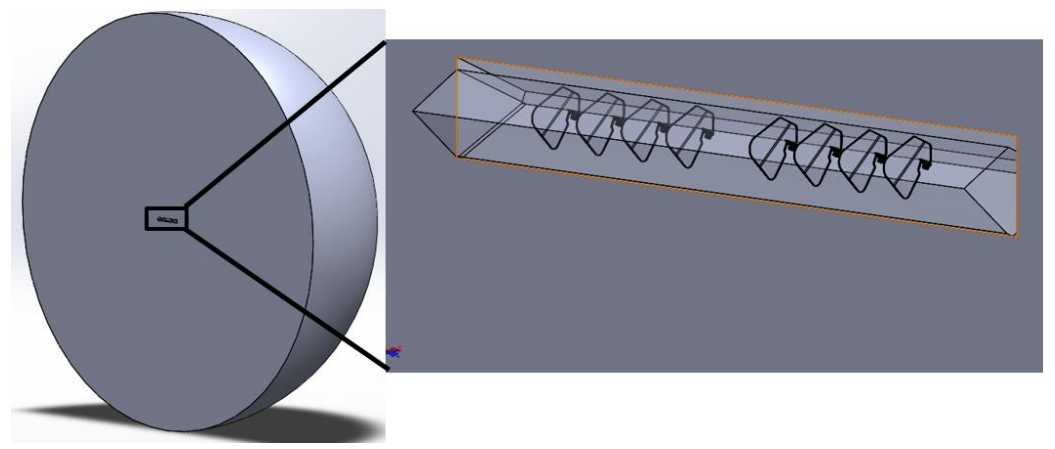

(a)

(b)

Fig. 2. (a) The half sphere of fluid domain and (b) location of vertical vane in transparent view.

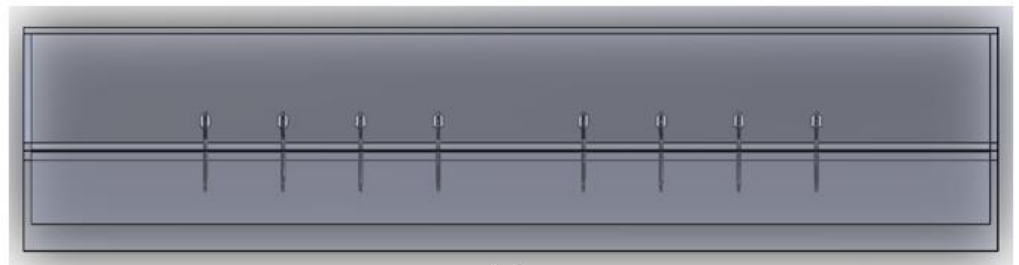

(a)

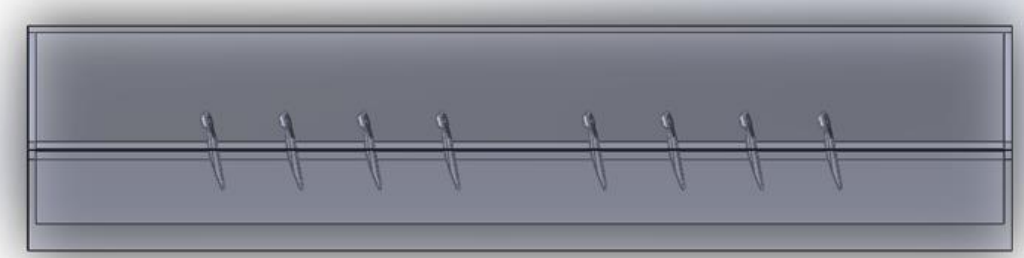

(b)

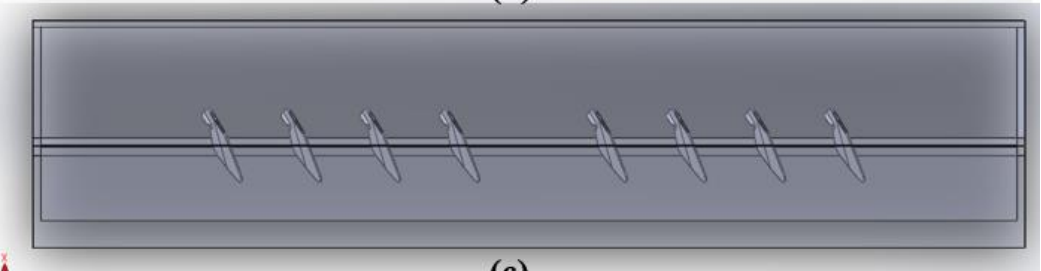

(c)

Fig. 3. Rotation of simplified vertical vane (a) $0^{\circ}$ (b) $15^{\circ}$ (c) $30^{\circ}$.

\subsubsection{Meshing generation}

The mesh quality of model is very important as it will affect the accuracy of solution generated in post-processing. First of all, unstructured type of mesh is applied for this model as the fluid domain has complicated geometry of vertical vane. By choosing the 
unstructured mesh, the meshing quality is improved and the computing time is also increased to solve for simulation [7].

Tetrahedral type of meshing element is selected due to the fillets and curvatures of vertical vanes and enclosed domain. After that, the edge sizing is added to shell of room air-conditioner. It is to increase the number of mesh element, so the velocity of air flow is captured with more accurate result. Moreover, inflation is added to the surfaces that bounded to the air flow inlet. The first layer thickness of $0.000643 \mathrm{~m}$ with 1.1 growth rate is set for the inflation. It is to ensure that the boundary layer effect is captured. The first layer of thickness is calculated based on the wall $\mathrm{Y}+$ value of 30 . The air flow that inside the boundary layer is named as viscous flow as it will form no slip condition where velocity of air flow equal to zero. Lastly, face meshing is added to the shell of room air-conditioner. It helps to organise the element equally over the surfaces.

However, meshing setup for $15^{\circ}$ is slightly different from that that. In order to reach the better convergence during post-processing, another inflation is added to the surfaces of vertical vanes. When the vertical vane is rotated to $15^{\circ}$ angle, it will cause more turbulent energy. Thus, mesh refinement is required to do for the surface of vertical vane. The meshing of model is shown in Fig. 4.

Although the meshing method applied to $15^{\circ}$ angle of simplified vertical vane is not same as $0^{\circ}$ angle of simplified vertical vane, it does not affect the selection of using same mesh size because meshing size is independent of grid refinement [7]. Hence, the meshing size will use the same after the grid independence.

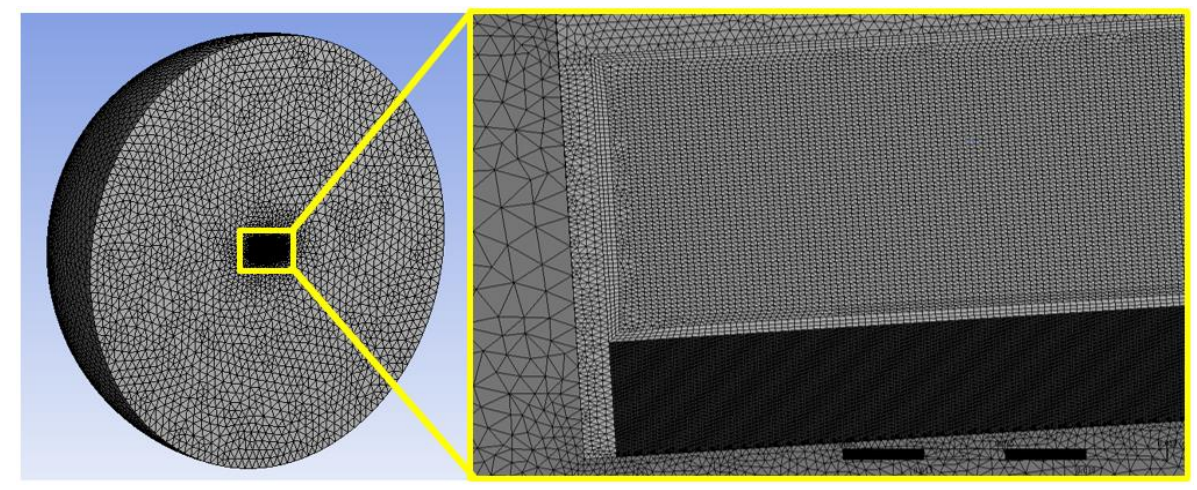

Fig. 4. The meshing of CFD model.

\subsubsection{Grid independence analysis}

Three level of meshing size is classified into coarse, medium and fine with respectively $0.008 \mathrm{~m}, 0.004 \mathrm{~m}$ and $0.002 \mathrm{~m}$ sizing. The purpose of doing grid independence analysis is to determine the maximum mesh size that can produce the same result as the smallest mesh size. The smallest mesh size for this model is $0.002 \mathrm{~m}$ as the element quality doesn't increase more after this value. The minimum growth rate for this mesh size is 1.7. Any value below 1.7 growth rate costs high increase in computing time of solving result. The advantage of doing grid independence analysis will save time on solving solution during post-processing.

As mentioned previously, $0^{\circ}$ angle of vertical vane is used as base reference. The following result presented in table is the result of base reference. From the results of Table 1 , it is not enough data to judge which mesh size to use. As the outlet velocity is the main 
focus in this study, the velocity of air flow should be measured and compared between these three different mesh sizes.

Table 1. Result of different mesh size

\begin{tabular}{|c|c|c|c|c|c|c|c|}
\hline Level & $\begin{array}{c}\text { Sizing } \\
\text { (m) }\end{array}$ & $\begin{array}{l}\text { Growth } \\
\text { rate }\end{array}$ & $\begin{array}{l}\text { Meshing } \\
\text { Quality }\end{array}$ & Node & Elements & Iteration & $\begin{array}{c}\text { Time } \\
\text { (hours) }\end{array}$ \\
\hline \multirow{2}{*}{ Coarse } & \multirow{2}{*}{0.008} & 1.15 & 0.80003 & 733666 & 3140164 & 1408 & 1.7 \\
\hline & & 1.17 & 0.79756 & 717586 & 3044681 & 597 & 0.6 \\
\hline \multirow{2}{*}{ Medium } & \multirow{2}{*}{0.004} & 1.15 & 0.80015 & 733224 & 3137247 & 2154 & 2.6 \\
\hline & & 1.17 & 0.79718 & 717363 & 3043687 & 831 & 1.0 \\
\hline \multirow{2}{*}{ Fine } & \multirow{2}{*}{0.002} & 1.15 & 0.79992 & 733761 & 3140411 & $>4000$ & $>10$ \\
\hline & & 1.17 & 0.79729 & 717637 & 3043217 & 770 & 0.9 \\
\hline
\end{tabular}

\subsubsection{Numerical setup}

RNG k- $\varepsilon$ turbulent model is derived from instantaneous Navier-Stokes equations or renormalization group which is using statistical technique. RNG k- $\varepsilon$ turbulent is applied to the CFD model because it has been proved to have good prediction for velocity of airflow at indoor environment. [6, 8]. Next, standard wall function is used for this conditions. For the outlet surface, it is defined as pressure outlet.

Lastly, the boundary conditions that have been set in ANSYS Fluent ver. 18 are shown in Table 2. Since the inlet velocity is $5.8 \mathrm{~m} / \mathrm{s}$ which is less than the $0.3 \mathrm{Ma}$ (Mach number), incompressible flow is applied. On the other hands, the volume of simplified vertical vane is 2.4 X 10-6 m3. This information is important to check whether the new design of vertical vane is minimised or not as compared to the simplified vertical vane.

Table 2. Boundary setting for CFD setup

\begin{tabular}{|c|c|c|c|c|}
\hline $\begin{array}{c}\text { Inlet } \\
\text { velocity } \\
(\mathbf{m} / \mathbf{s})\end{array}$ & $\begin{array}{c}\text { Temperature } \\
\left(\mathbf{(}^{\mathbf{}} \mathbf{C}\right)\end{array}$ & $\begin{array}{c}\text { Density } \\
\text { of air } \\
\left(\mathbf{k g} / \mathbf{m}^{\mathbf{3}}\right)\end{array}$ & $\begin{array}{c}\text { Kinematic } \\
\text { viscosity }\end{array}$ & $\begin{array}{c}\text { Viscosity } \\
\text { of air } \\
(\mathbf{k g} / \mathbf{m s})\end{array}$ \\
\hline 5.8 & 20 & 1.205 & $1.511 \times 10^{-5}$ & 1.82076 \\
\hline
\end{tabular}

\subsubsection{Assumptions}

The assumptions of this research's study have been made as following statements:

i. The velocity inlet for the room air-conditioner is uniform and constant at the inlet surface.

ii. The temperature of room which is the enclose domain is constant.

iii. No backflow is occurred at the end of wall surface (outlet).

\subsubsection{Calculation}

The method of analysing simplified vertical vane will be defined by calculating the distance of how far the airflow can be achieved. First of all, any velocity of airflow that is below $0.25 \mathrm{~m} / \mathrm{s}$ in velocity contours is considered no wind according to the standard requirement from Panasonic. From this point, the distance where the velocity of airflow is higher than and equal to $0.25 \mathrm{~m} / \mathrm{s}$ will be taken into calculation.

Furthermore, simulation result of different angle of vertical vane is analysed for efficiency of how accurate the direction of airflow coming out from vertical vane. The distance, where velocity of airflow is equal to and higher than $0.25 \mathrm{~m} / \mathrm{s}$, in $z$-axis is taken 
into calculation by applying trigonometry method. For distance X, it is measured $2 \mathrm{~m}$ away from the room air-conditioner. For the measured line of starting point will be located at $1.13 \mathrm{~m}$ ( $\mathrm{Y}$ axis) below the room air-conditioner. The calculation of angle of wind is illustrated in Fig 5.

$$
\tan \theta_{\text {wind }}=\frac{\text { distance in } z \text { axis (velocity } \geq 0.25 \mathrm{~m} / \mathrm{s} \text { ) }}{\text { distance in } x \text { axis (velocity } \geq 0.25 \mathrm{~m} / \mathrm{s} \text { ) }}
$$

The efficient of wind direction is defined as equation below.

$$
\text { Efficiency of wind direction }=\frac{\theta_{\text {wind }}}{\theta_{\text {vertical vane }}} \times 100 \%
$$

where $\theta_{\text {wind }}$ is the angle of wind in simulation and $\theta_{\text {vertical vane }}$ is the angle of rotation of vertical vane inside room air-conditioner.

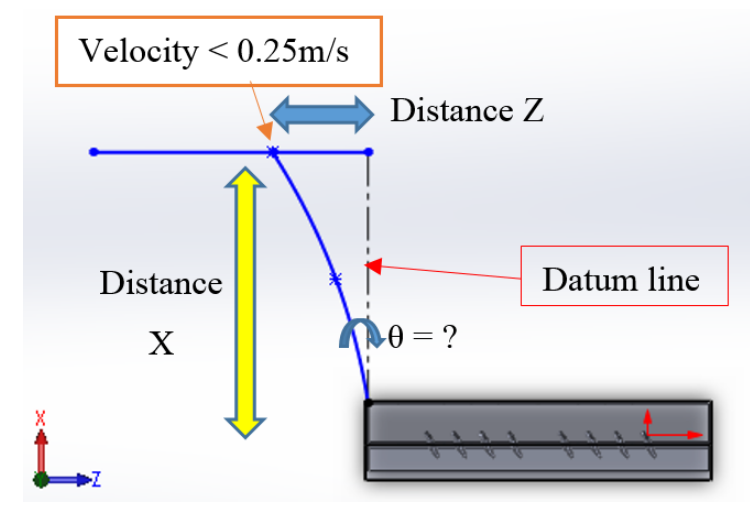

Fig. 5. Calculation of angle of wind.

The equation of efficiency of wind direction is used to define the efficiency of dissipating cold air. It can be applied as long as the location line is within the pathway of airflow in simulation result. In order to make the efficiency of wind direction is comparable with different angles and new designs of vertical vane; the location of measured line is fixed for all case. The location of measured line that determines the velocity of airflow from starting point to ending point is shown in Table 3.

Table 3. Distance from datum line

\begin{tabular}{|c|c|c|c|}
\hline Location & $\begin{array}{c}\boldsymbol{x} \text {-axis } \\
(\mathbf{m})\end{array}$ & $\begin{array}{c}\boldsymbol{y} \text {-axis } \\
(\mathbf{m})\end{array}$ & $\begin{array}{c}\boldsymbol{z} \text {-axis } \\
(\mathbf{m})\end{array}$ \\
\hline Starting point & 2 & -1.13 & 0 \\
\hline Ending point & 2 & -1.13 & -1.5 \\
\hline
\end{tabular}

\section{Result and discussion}

\subsection{Grid independence analysis}

The result of $0^{\circ}$ angle of vertical vane simulated from $0.002 \mathrm{~m}, 0.004 \mathrm{~m}$ and $0.008 \mathrm{~m}$ mesh size is analysed. The velocity of airflow along the boundary edge and line 1 are measured. The measured location is shown in Fig. 6 and the graph of velocity against the location is plotted in Fig. 7. Observing the graph from Fig. 7, the fine mesh size has higher velocity 
along the boundary edge as compared to medium and coarse mesh size. However, the velocity result generated by medium mesh size is more reasonable. The reason is the velocity at the middle of boundary edge reached uniform velocity of airflow where this location has big spacing between each vertical vane. Thus, the velocity of airflow is expected to have higher velocity compared to other location. Besides that, the velocity of airflow is almost symmetrical. The medium mesh size can be chosen as constant variable for meshing setting.

In additions, the trend line of medium mesh has the same behaviour as the fine mesh in Fig 8. For the coarse mesh, it only has same pattern of trend line at left side of line 1 . Hence, the grid independence analysis is done and the medium mesh which is $0.004 \mathrm{~m}$ is used as constant mesh size to all case studies. Lastly, the growth rate of 1.7 is selected as constant variable since its result is almost same as result generated with 1.5 growth rate.

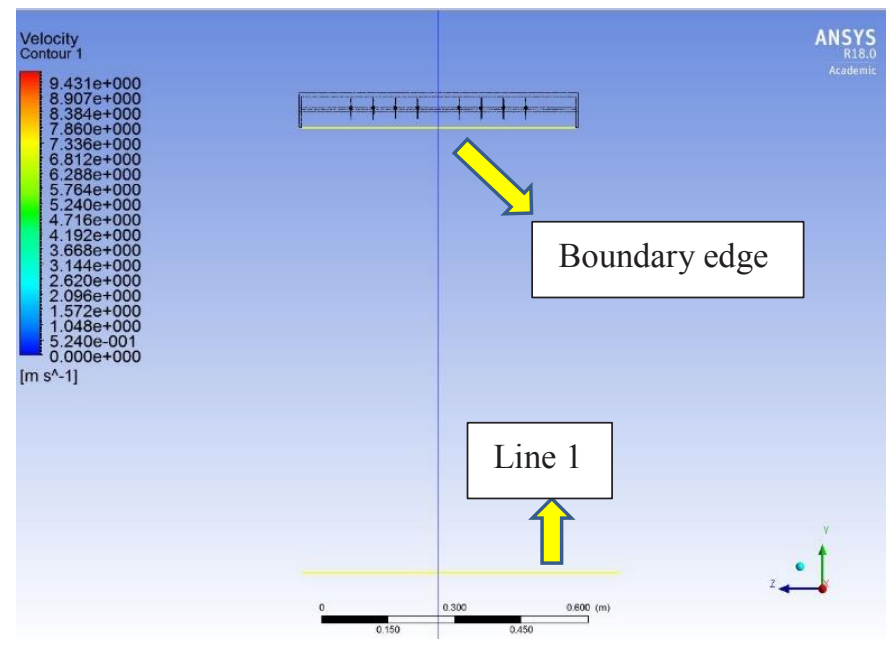

Fig. 6. Location of boundary edge and line 1.

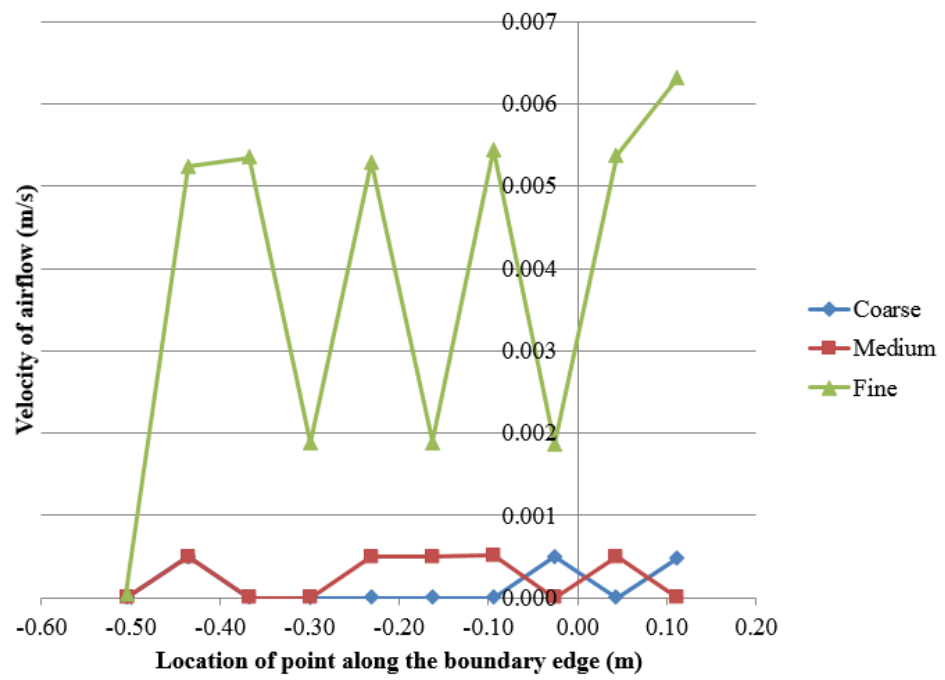

Fig. 7. Graph of velocity against location of point for different mesh size. 


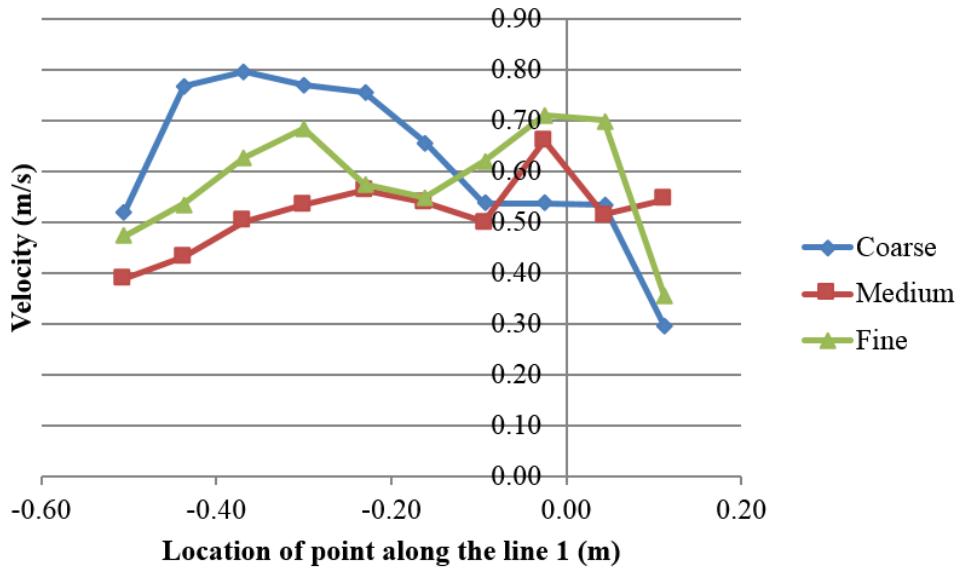

Fig. 8. Graph of velocity against location of point for different mesh size.

\subsection{Efficiency of wind direction}

The velocity streamline of $15^{\circ}$ and $30^{\circ}$ of vertical vane is plotted in Fig. 9. Besides that, the result of efficiency of wind direction is calculated and shown in Table 4. Comparing efficiency of wind direction of $15^{\circ}$ and $30^{\circ}$ of vertical vane, it can be concluded that the efficiency of wind direction is decreased as the angle of vertical vane increased. This result explained the reason why the angle of vertical vane of this room air-conditioner has been set a limit to rotate. The higher the angle of vertical vane to rotate the lesser the efficiency of wind direction and dissipating cold air. These result will used as based reference in order to check whether new designs of vertical vane has made improvement on it.

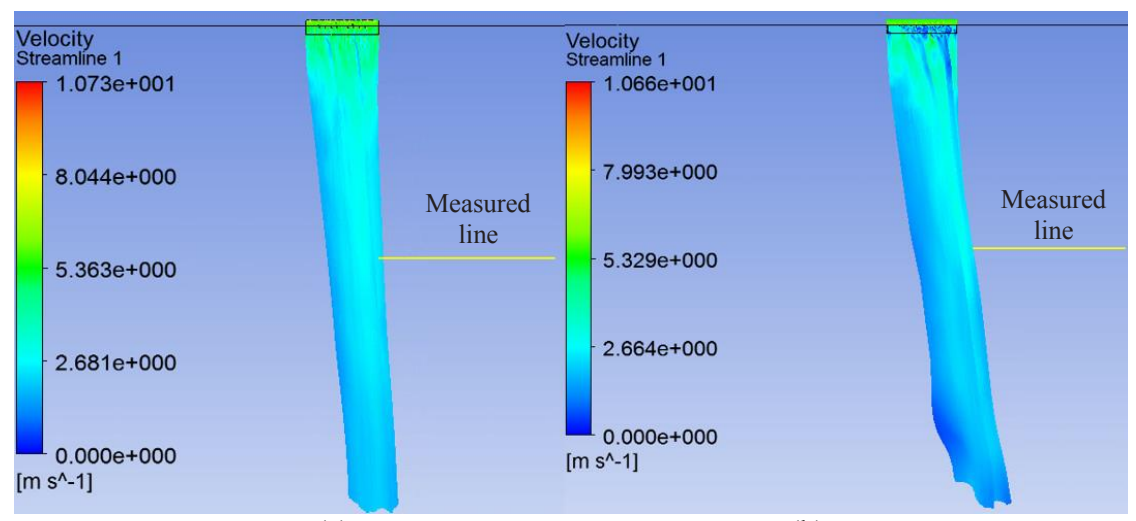

(a)

(b)

Fig. 9. Velocity streamline of vertical vane (a) $15^{\circ}$ and (b) $30^{\circ}$.

Table 4. Efficiency of wind direction

\begin{tabular}{|c|c|c|c|c|}
\hline $\begin{array}{c}\text { Angle of rotation of } \\
\left.\text { vertical vane } \mathbf{(}^{\mathbf{(}}\right)\end{array}$ & $\begin{array}{c}\text { Distance } \mathbf{Z} \\
(\mathbf{m})\end{array}$ & $\begin{array}{c}\text { Distance X } \\
\mathbf{( m )}\end{array}$ & $\begin{array}{c}\text { Angle of wind } \\
(\mathbf{(})\end{array}$ & $\begin{array}{c}\text { Efficiency of } \\
\text { wind direction (\%) }\end{array}$ \\
\hline 15 & 0.30503 & 2.0 & 8.67 & 57.81 \\
\hline 30 & 0.50839 & 2.0 & 14.26 & 47.54 \\
\hline
\end{tabular}


The velocity contours at mid-plane of vertical vane are shown in Figs. 10 and 11. Based on these figures, it proved that the higher the angle of rotation of vertical vane will lead to shorter distance of the airflow can be achieved at that angle. It also explained $100 \%$ efficiency of wind direction can't be obtained since the minimum velocity of airflow, 0.25 $\mathrm{m} / \mathrm{s}$ is indicated before reaching the measured line.

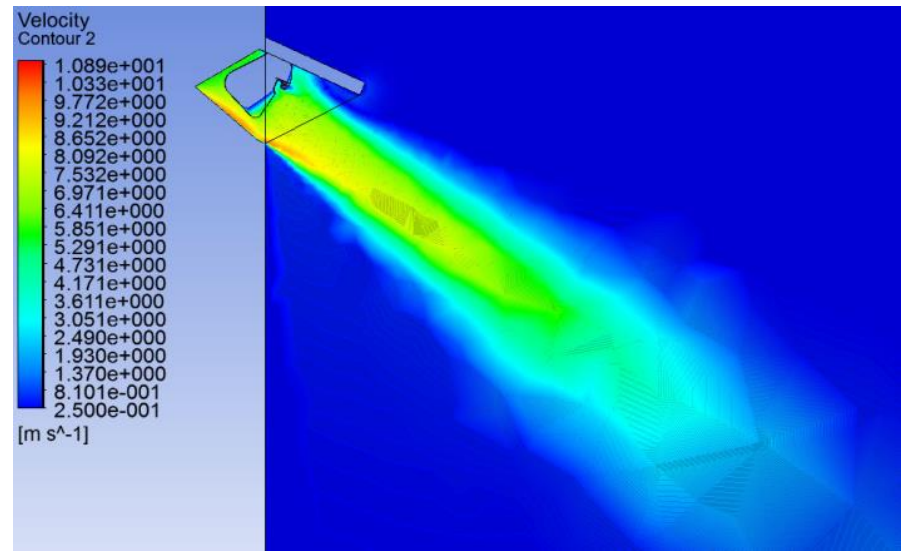

Fig. 10. Velocity contour at mid-plane of $15^{\circ}$ of vertical vane.

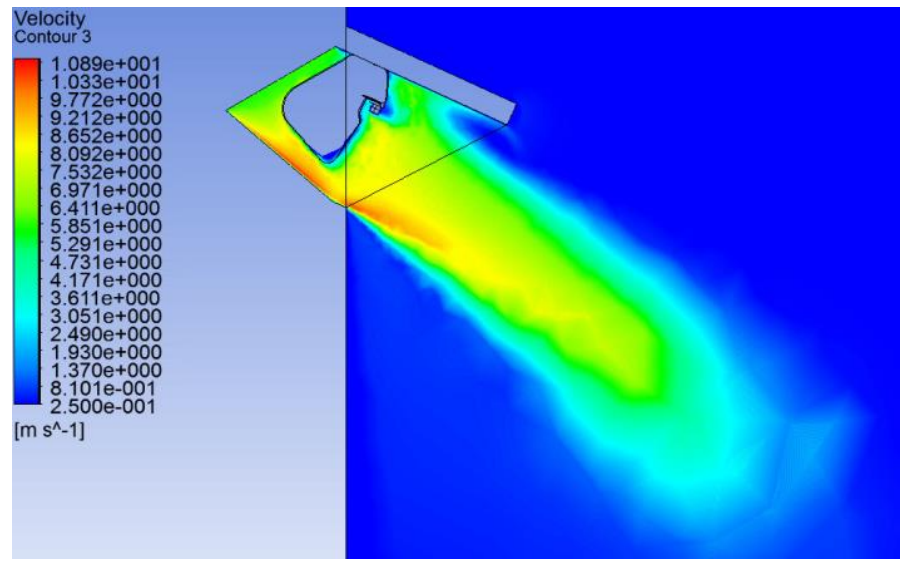

Fig. 11. Velocity contour at mid-plane of $30^{\circ}$ of vertical vane.

\subsection{Different contour analysed at $30^{\circ}$ of vertical vane}

The pressure contour at mid-plane of $30^{\circ}$ of vertical vane is plotted and shown in Fig. 12. It is used to investigate which parts of vertical vane are main parameters of affecting the efficiency of wind direction. According to the Fig. 12, high increase in gauge pressure is clearly determined at leading edge of vertical vane compared to Fig. 11. Based on Bernoulli's principle, increase in velocity will cause decrease in pressure [4]. In this study, the low velocity of airflow at leading edge induces the high increase in pressure of airflow.

Another velocity contour in Fig. 13 is plotted perpendicular to the inlet and is aligned to leading edge of vertical vane. It is plotted to observe how the velocity of airflow varies when airflow is passing the vertical vane. Referring to the top view of velocity contour, that perpendicular to inlet in Fig. 14, the velocity of airflow passing by the left surface of vertical vane is lower than the velocity of airflow at right surface of vertical vane. In 
additions, the rear part of vertical vane induce vortex. Vortex induced at rear part of vertical vane caused decreased in velocity of airflow.

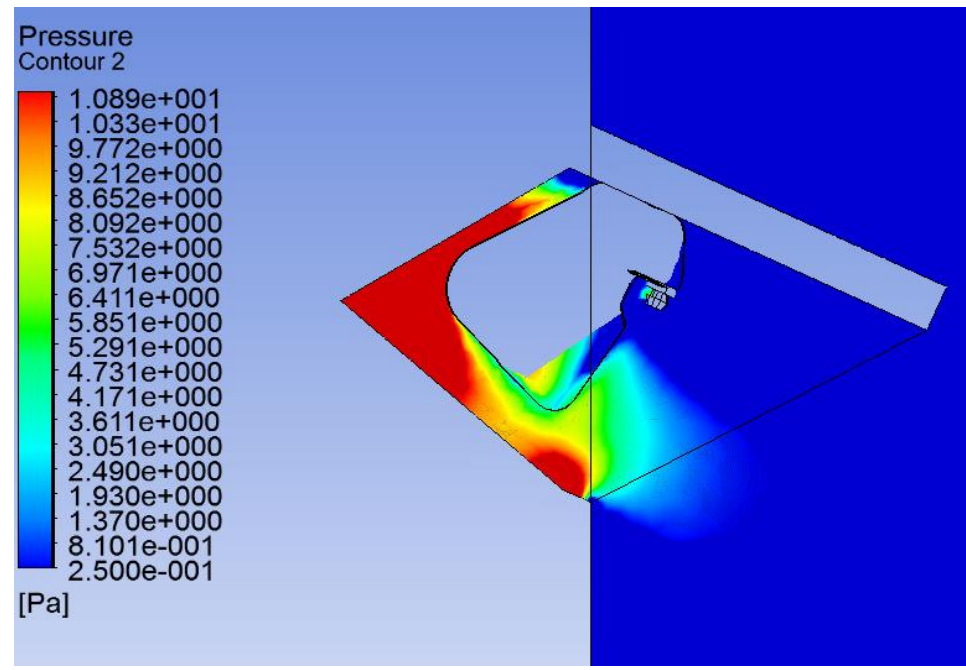

Fig. 12. Pressure contour at mid-plane of $30^{\circ}$ of vertical vane.

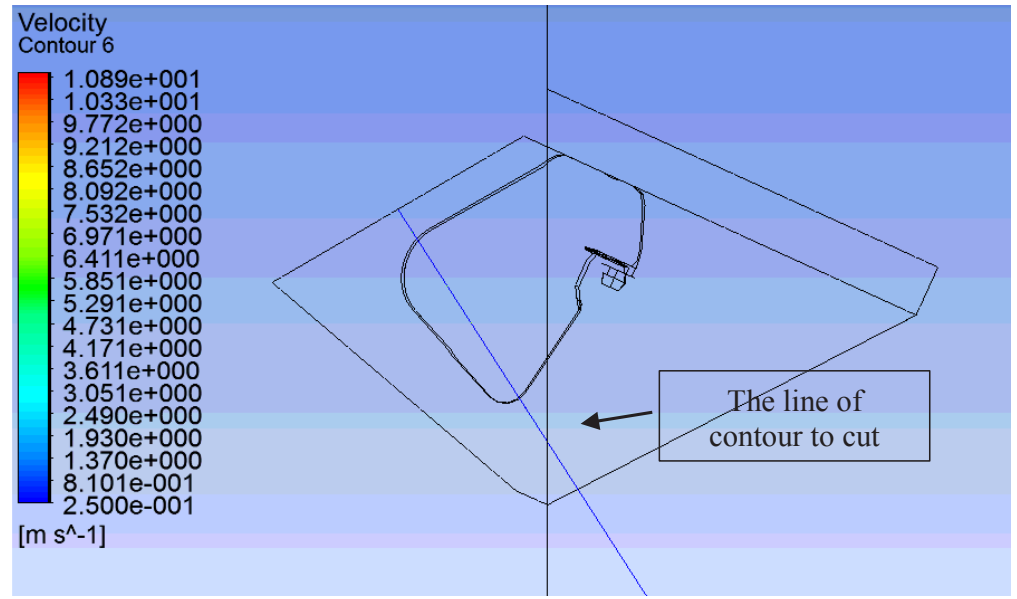

Fig. 13. Position of velocity contour cut from inlet.

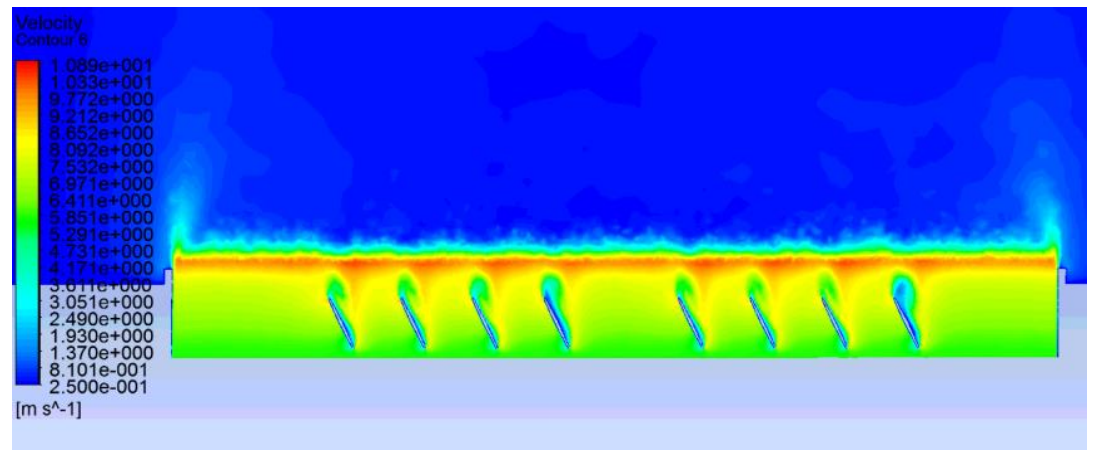

Fig. 14. Top view of the velocity contour cut perpendicular to inlet 
Thus, two parameters are selected to study for geometry optimisation of vertical vane. The parameters studies will be the length of span and length of tip chord. The name of length is shown in Fig. 15. The span length and tip chord length of simplified vertical vane are $0.04436 \mathrm{~m}$ and $0.04478 \mathrm{~m}$ respectively.

The restriction set for parameter studies are as following:

1. The surface of root chord that is attached to room air-conditioner is constant.

2. The total height of vertical vane is maximum length. (due to size of product)

3. The total area of vertical vane is constant.

4. The material of vertical vane is constant.

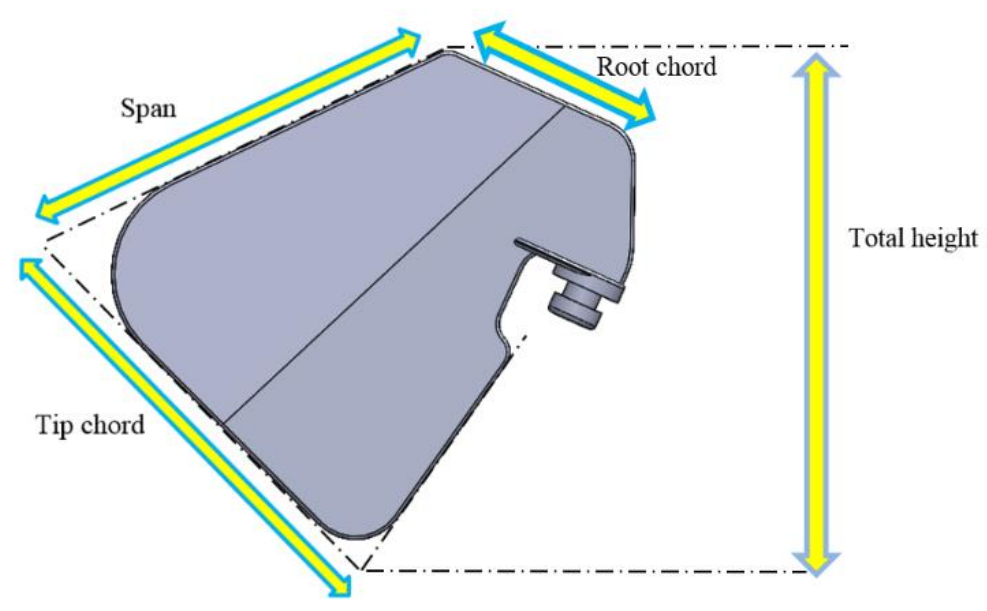

Fig. 15. Name of different length of vertical vane.

\subsection{Parametric studies for geometry optimisation of vertical vane}

Since long span, tip chord and short span are chosen as main parameter studies, new designs of vertical vane are drawn based on 5\%,10\% and $15 \%$ decreased in vane surface area. A basic sketching is illustrated in Fig. 16 shown position of each parameter study with different percentage decreased in vane surface area.

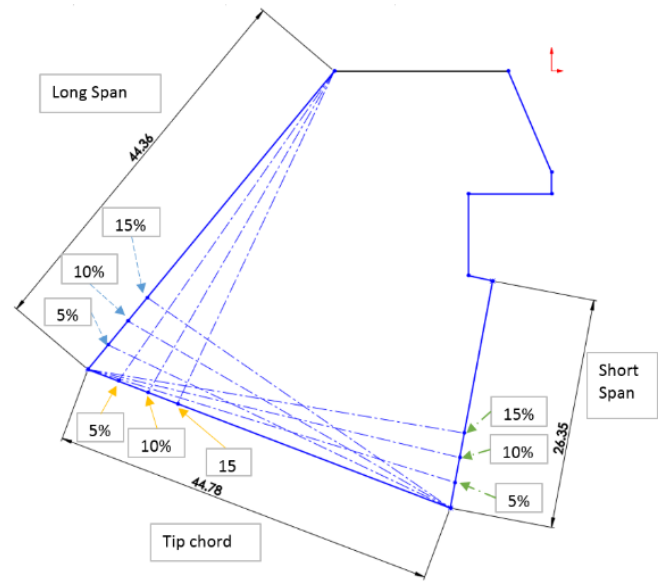

Fig. 16. The position of each parameter study with different percentage decreased in vane surface area 
In this paper, the simulation is only run for one side (turning left side) of direction because the design of CFD model is symmetrical with respect to centre of shell of room airconditioner. Moreover, only 15 degree angle of vertical vane is studied as it is sufficient to identify which parameter is important to analyse. Furthermore, the result of new vertical vane can be compared with base reference (efficiency of $15^{\circ}$ and $30^{\circ}$ of simplified vertical vanes). In previous discussion, it is found that the higher the angle of vertical vane, the lower the efficiency of wind direction. As long as the result of new vertical vane has same trend as base reference, the reliability of simulation result is justified. The CAD drawing of new vertical vane based on each parameter study at different percentage decreased in vane surface area is shown in Fig 16. The simulation result of new vertical vane is tabulated in Table 5.

According to the Table 5, the changes in efficiency of wind direction for long span are significantly small. Besides that, the trend of decreasing the vane surface area of long span gives result of dropping in efficiency of wind direction. Hence, it can be assumed that long span can't be decreased but it could be increased for improving efficiency of wind direction.

Next, all the result of short span shows negative impact to efficiency of wind direction. So, it is better to keep length of short span as constant as it can't be exceed the length of total height.

Lastly, the result of $5 \%$ and $10 \%$ decreased in vane surface area at tip chord show negative impact to efficiency of wind direction. However, the $15 \%$ decreased in vane surface area at tip chord is actually higher than the efficiency of base reference which is $57.81 \%$. The design of this new vertical vane is selected best configuration of design since its efficiency of wind direction is $60.32 \%$ which is increased by $2.51 \%$ as compared to efficiency of base reference.

Table 5. Result of each parameter study at different percentage decreased in vane surface area

\begin{tabular}{|c|c|c|c|c|c|c|}
\hline \multirow{2}{*}{$\begin{array}{c}\text { Parameter } \\
\text { studied }\end{array}$} & $\begin{array}{c}\text { Percentage } \\
\text { decreased } \\
\text { in vane } \\
\text { surface } \\
\text { area (\%) }\end{array}$ & $\begin{array}{c}\text { Distance } \\
\text { from fix } \\
\mathbf{p o i n t} \\
\mathbf{( m m )}\end{array}$ & $\begin{array}{c}\text { Distance } \\
\mathbf{Z}(\mathbf{m})\end{array}$ & $\begin{array}{c}\text { Distance } \\
\mathbf{X}(\mathbf{m})\end{array}$ & $\begin{array}{c}\text { Angle } \\
\mathbf{o f} \\
\text { wind } \\
\mathbf{(} \mathbf{})\end{array}$ & $\begin{array}{c}\text { Efficiency } \\
\text { of wind } \\
\text { direction } \\
\mathbf{( \% )}\end{array}$ \\
\hline \multirow{3}{*}{ Long Span } & 5 & 3.6480 & 0.27601 & 2 & 7.86 & 52.38 \\
\cline { 2 - 7 } & 10 & 7.2020 & 0.27146 & 2 & 7.73 & 51.53 \\
\cline { 2 - 7 } & 15 & 10.6955 & 0.26691 & 2 & 7.60 & 50.68 \\
\hline \multirow{3}{*}{ Tip chord } & 5 & 3.7599 & 0.26236 & 2 & 7.47 & 49.82 \\
\cline { 2 - 7 } & 10 & 7.4350 & 0.28966 & 2 & 8.24 & 54.94 \\
\cline { 2 - 7 } & 15 & 11.0610 & 0.31847 & 2 & 9.05 & 60.32 \\
\hline \multirow{3}{*}{ Short Span } & 5 & 2.9729 & 0.20776 & 2 & 5.93 & 39.54 \\
\cline { 2 - 7 } & 10 & 5.8646 & 0.22293 & 2 & 6.36 & 42.40 \\
\cline { 2 - 7 } & 15 & 8.6985 & 0.21231 & 2 & 6.06 & 40.40 \\
\hline
\end{tabular}

\section{Conclusions}

The efficiency of wind direction for different angles for vertical vane is studied. The efficiency of wind direction for $15^{\circ}$ of vertical vane is $57.81 \%$ while the efficiency of wind direction for $30^{\circ}$ is $47.54 \%$. The results of the efficiency of wind direction are used as base reference. The parameters studied for optimisation of vertical vane are focused at length of long span, tip chord and short span. The design of $15 \%$ decreased in vane surface area at tip chord is the best optimised design of vertical vane because the efficiency of wind direction is the highest as $60.32 \%$. In addition to that, the mass of vertical vane of this design is lower than base reference and thus it is optimised without bringing any negative effect to the efficiency of dissipating cold. 


\section{References}

1. B. Xia, D.-W., Sun, Comput. Electron. Agric. 34 (1-3) 5-24 (2004)

2. T. Nakanishi, T. Nakamura, W. Youichirou, H. Katsumasa, K. Takahiro, Technical Paper, 53(160), 1-9 (2007)

3. M. H. M. Noh, H. Rashid, A. H. A. Hamid, and M. F. Iskandar, Procedia Eng, 41, 1761-1768 (2012).

4. Y. A. Cengel, J. M.Cimbala. Fluid mechanics: Fundamentals and applications, $\left(3^{\text {rd }}\right.$ Ed. McGraw-Hill 2014)

5. B. S. Hurak, Undergraduate Honors Thesis, The Ohio State University (2011)

6. T. Karimipanah, H. B. Awbi, M. Sandberg, C. Blomqvist, Build. Environ, 42(2) 647655 (2007)

7. N. Mao, M. Song, S. Deng, Appl. Energy 180, 536-545 (2016)

8. S. Lin, B. T. Tee, C. F. Tan, 7th Int. Conf. on Cooling \& Heating Technologies, Bristol, United Kingdom (2015)

9. S. Sadrizadeh, S. Holmberg, Proceedings of 9th International Conference on Indoor Air Quality Ventilation \& Energy Conservation in Buildings, 1-7 (2016) 